\title{
Survey of "Do not resuscitate" orders in a district general hospital
}

\author{
Emma J Aarons, Nicholas J Beeching
}

\section{Abstract}

Objective-To evaluate the local use of written "Do not resuscitate" orders to designate inpatients unsuitable for cardiopulmonary resuscitation in the event of cardiac arrest.

Design-Point prevalence questionnaire survey of inpatients' medical and nursing records.

Setting -10 acute medical and six acute surgical wards of a district general hospital.

Participants-Questionnaires were filled in anonymously by nurses and doctors working on the wards surveyed.

Main outcome measures-Responses to questionnaire items concerning details about each patient, written orders not to resuscitate in the medical case notes and nursing records, whether prognosis had been discussed with patients' relatives, whether a "crash call" was perceived as appropriate for each patient, and whether the "crash team" would be called in the event of arrest.

Results-Information was obtained on 297 (93.7\%) of 317 eligible patients. Prognosis had been discussed with the relatives of 32 of 88 patients perceived by doctors as unsuitable for resuscitation. Of these 88 patients, 24 had orders not to resuscitate in their medical notes, and only eight of these had similar orders in their nursing notes.

Conclusions - In the absence of guidelines on decisions about resuscitation, orders not to resuscitate are rarely included in the notes of patients for whom cardiopulmonary resuscitation is thought to be inappropriate. Elective decisions not to resuscitate are not effectively communicated to nurses. There should be more discussion of patients' suitability for resuscitation between doctors, nurses, patients, and patients' relatives. Suitability for resuscitation should be reviewed on every consultant ward round.

\section{Introduction}

Studies of the outcome of cardiopulmonary resuscitation of patients who suffer a cardiac arrest in hospital shows that only $8 \cdot 7-14 \cdot 0 \%$ are discharged alive. ${ }^{1-4}$ Characteristics of patients before arrest that predict death in hospital include a homebound lifestyle, cancer, hypotension, and renal failure. ${ }^{1.3}$ With or without specific knowledge of these risk factors for mortality, most doctors and nurses believe that there are some patients for whom resuscitation in the event of cardiopulmonary arrest would be inappropriate for reasons of futility or poor quality of life. Clinical teams can exclude these patients from resuscitation attempts by means of "Do not resuscitate" orders communicated to nurses, who otherwise would call the "crash team."

Fazakerley Hospital is a district general hospital with 430 acute medical and surgical beds. In September 1990 the monthly audit meeting of the medical division brought attention to the absence of a formal hospital policy on "Do not resuscitate" decisions and the apparent frequency of inappropriate calls to the crash team after a cardiac arrest. A survey of inpatients' medical and nursing records was proposed in order to find out how effectively written orders not to resuscitate were being used.

\section{Methods}

A point prevalence survey of medical and nursing inpatient records was carried out by means of a questionnaire. The questionnaire was sent to each of the 10 acute medical and six acute surgical wards. Obstetric and gynaecology wards were excluded. A covering letter requested that the questionnaire should be filled in anonymously and at the earliest opportunity by the nurse in charge and by the junior doctor(s) looking after the patients. Every patient on the ward at that time was to be entered on the questionnaire.

The questionnaire comprised two sections. In the first section the nurse in charge was asked, for each patient, whether he or she would call the crash team in the event of a cardiopulmonary arrest, whether he or she thought a "crash call" appropriate, and whether "Not for resuscitation" or similar was noted in the nursing records. In the second section the doctor was asked to enter details of each patient's age, sex, date of admission, diagnoses, expected survival, cognitive function, and mobility; whether the patient's prognosis had been discussed with his or her relatives; the date and content of any order not to resuscitate in the medical notes; and whether he or she thought a crash call appropriate. No attempt was made to validate the answers by repeating the questionnaire on a different day.

The number of patients available for inclusion in the survey was determined by obtaining each ward's bed occupancy figure for the day on which the questionnaire was filled in.

The study was approved by the heads of the medical and surgical divisions and by the unit nurse manager. Data were tabulated and analysed by using a public domain software package (Epi Info 5, Centers for Disease Control, Atlanta).

\section{Results}

All 16 questionnaires were filled in and returned. Information was obtained on $297(93.7 \%)$ of the 317 patients on the wards at the time the questionnaires were filled in. Their average age was $67 \cdot 1$ years (SD $15.6)$, and $167(56 \cdot 2 \%)$ were female. Their median length of stay up to the time of the survey was eight days. Eighty four $(28.3 \%)$ were under the care of geriatricians, and $106(35.7 \%)$ were patients in surgical specialties (urology, general surgery, orthopaedics, thoracic surgery). Twelve $(4 \cdot 0 \%)$ patients were on a ward of specialty other than their own. Forty nine $(16.5 \%)$ of the patients had cancer. Ten $(3.4 \%)$ were expected to survive less than one month. Thirteen $(4 \cdot 4 \%)$ patients were said to have very poor cognitive function, and $27(9 \cdot 1 \%)$ were bedridden.

Cardiopulmonary resuscitation was thought by doctors to be inappropriate for $88(29.6 \%)$ of the 297 patients surveyed, but for only four $(3 \cdot 8 \%)$ of the surgical patients. None of 10 patients expected to survive less than one month was regarded as suitable for resuscitation. Resuscitation was thought to be
Dr Beeching.

BMF 1991;303:1504-6 
inappropriate for $25(92 \cdot 6 \%)$ bedridden patients and 12 $(92.3 \%)$ of the patients with very poor cognitive function. Thirty four $(69.4 \%)$ patients with cancer were seen as suitable for resuscitation. Table I shows the relation between ages of patients and the perceived suitability for resuscitation.

TABLE I-Relation between age of patients and suitability for cardiopulmonary resuscitation as perceived by doctors. Figures are numbers of patients

\begin{tabular}{cccr}
\hline $\begin{array}{l}\text { Age group } \\
\text { (years) }\end{array}$ & $\begin{array}{c}\text { Suitable for } \\
\text { resuscitation }\end{array}$ & $\begin{array}{c}\text { Not suitable for } \\
\text { resuscitation }\end{array}$ & Total \\
\hline $0-9$ & 0 & 0 & 0 \\
$10-19$ & 3 & 0 & 3 \\
$20-29$ & 8 & 0 & 8 \\
$30-39$ & 11 & 0 & 11 \\
$40-49$ & 15 & 0 & 15 \\
$50-59$ & 30 & 5 & 35 \\
$60-69$ & 59 & 16 & 75 \\
$70-79$ & 51 & 29 & 80 \\
$80-89$ & 23 & 15 & 46 \\
$90-99$ & 1 & 88 & 16 \\
\hline Total & $201^{\star}$ & & 289 \\
\hline
\end{tabular}

^Eight patients were excluded because age was omitted from questionnaire.

Orders not to resuscitate were written in the medical notes of only $24(27 \cdot 3 \%)$ patients thought by doctors to be unsuitable for resuscitation and for only eight $(17 \cdot 8 \%)$ such patients in the specialty of geriatrics. Orders were also present in the notes of two patients regarded as suitable for resuscitation. Of the 26 orders, the contents of five were not recorded on the questionnaires. Twenty stated "Not for resuscitation," "NFR," "Not for 2222" (the telephone number for a cardiac arrest call), or "Resuscitation would be inappropriate." One stated "Tender loving care only." The median period since the order had been written was 11 days.

Of the 26 patients who had a "Do not resuscitate" order in their case notes, only $10(38.5 \%)$ also had such an order in their nursing records. Nurses' responses to the question "Would you call the crash team if this patient had an arrest?" indicated that none of these 10 would have had the crash team called had they had an arrest. In two cases because the patients had unrescinded orders in their medical and nursing notes the crash team apparently would not have been called even though both the doctor and the nurse regarded resuscitation as appropriate (table II). Conversely, crash calls would have been made for 24 patients despite both the doctor and the nurse perceiving them to be unsuitable for resuscitation (table II). Of 16 patients thought by the doctors to be unsuitable for resuscitation and who had an order not to resuscitate in their medical notes alone, eight apparently would have had the crash team called (table III).

Prognosis was known to have been discussed with the relatives of 69 of $203(34 \cdot 0 \%)$ patients, but a note of this discussion had been made in only $31(44.9 \%)$ cases. Prognosis had been discussed with the relatives of only $32(36.4 \%)$ patients thought to be unsuitable for resuscitation, $21(42 \cdot 9 \%)$ patients with cancer, and six of the 10 patients expected to survive less than one month.

TABLE II-Concordance of doctors' and nurses' perceptions of patients' suitability for resuscitation, tabulated by whether or not attending nurse would call crash team. Figures are numbers of patients

\begin{tabular}{lrrr}
\hline & \multicolumn{3}{c}{$\begin{array}{c}\text { Would nurse call } \\
\text { crash team? }\end{array}$} \\
\cline { 2 - 3 } Views on resuscitation & \multicolumn{1}{c}{ Yes } & No & Total \\
\hline Doctor and nurse in favour of resuscitation & 186 & 2 & 188 \\
Only doctor in favour of resuscitation & 9 & 5 & 14 \\
Only nurse in favour of resuscitation & 22 & 1 & 23 \\
Doctor and nurse against resuscitation & 24 & 41 & 65 \\
\hline Total & 241 & 49 & $290^{\star}$ \\
\hline
\end{tabular}

$\star$ Views not recorded for seven patients.
TABLE III-Concordance of medical and nursing orders not to resuscitate among 88 patients perceived by doctors as unsuitable for resuscitation, tabulated by whether or not attending nurse would call crash team. Figures are numbers of patients

\begin{tabular}{lrrr}
\hline & \multicolumn{3}{c}{$\begin{array}{c}\text { Would nurse call } \\
\text { crash team? }\end{array}$} \\
\cline { 2 - 3 } Orders not to resuscitate present & \multicolumn{1}{c}{ Yes } & No & Total \\
\hline In neither medical nor nursing notes & 38 & 24 & 62 \\
In medical notes only & 8 & 8 & 16 \\
In nursing notes only & 0 & 2 & 2 \\
In both medical and nursing notes & 0 & 8 & 8 \\
\hline Total & 46 & 42 & 88
\end{tabular}

\section{Discussion}

"Do not resuscitate" orders were written in the medical notes of only a minority of patients perceived to be unsuitable for resuscitation. However, when orders were written they were clear. This contrasts favourably with the findings of a recent national survey of Swedish hospital physicians, in which 191 symbols and 31 code words were reported as being used for "Do not resuscitate."'

Communication to nurses of doctors' perceptions of patients' unsuitability for resuscitation was extremely poor. Even when orders were written in the medical notes the nurses often did not know. Meanwhile, some patients without orders not to resuscitate in either their medical or their nursing notes but thought by the nurses to be unsuitable for resuscitation apparently would not have had the crash team called. We could not validate nurses' responses to the question of whether the crash team would be called, but we note that in the absence of a written order not to resuscitate, not calling the crash team could place a nurse in an indefensible medicolegal position. ${ }^{6}$ When these orders had been written in the nursing records nurses were invariably aware of them and declared their intention to act on them

There is no independent relation between age and outcome of attempted resuscitation, but a homebound lifestyle, cancer, hypotension, renal failure, and pneumonia all predict unsuccessful resuscitation. ${ }^{1-4}$ We hope that the apparent association noted in this study between age and perceived inappropriateness of resuscitation arose because older people were more likely to have multiple diseases and impaired functional capacities. Decisions to withhold resuscitation in the event of a cardiopulmonary arrest should be based on knowledge of characteristics before arrest in relation to outcome, and there is a need for routine collection, analysis, and dissemination of such information. ${ }^{1}$ Meanwhile, decisions on resuscitation should not be made unilaterally. The diversity of perspectives and opinions of consultants, junior doctors, nurses, patients' relatives, and patients themselves can all make valuable contributions.?

This survey showed that prognosis, let alone resuscitation in the event of arrest, was rarely discussed with patients' relatives. In the United States discussion of resuscitation with patients or their relatives, or both, occurs more frequently. ${ }^{8.10}$ Indeed, where legislation on resuscitation exists such a discussion is a precondition for writing "Do not resuscitate" orders. ${ }^{910}$

Stolman et al have recently surveyed attitudes to resuscitation at a suburban hospital in New Jersey." They found that $65(67 \%)$ patients wanted to participate in decisions and that $64(66 \%)$ patients did not think discussing resuscitation was cruel and insensitive. Nevertheless, 18 of 60 physicians questioned felt uncomfortable discussing resuscitation with patients. In the United Kingdom such discussions may be left to the most junior medical staff, yet these people do not feel equipped enough. ${ }^{12}$ 
The ethical issues surrounding orders not to resuscitate are complex and increasingly a matter for legislative acts and judicial decisions. ${ }^{113}$ Furthermore, decisions on resuscitation have implications for resource management. Despite these issues "Do not resuscitate" orders in many British hospitals are used without guidelines and without assessment of their effectiveness in preventing futile cardiopulmonary resuscitation.

This survey indicates that in the absence of local guidelines about decisions on resuscitation many crash calls may be inappropriate. We advocate more discussion of patients' suitability for resuscitation between doctors, nurses, patients, and patients' relatives. We suggest that the most senior available doctor of the admitting team should be responsible for making initial resuscitation decisions, writing these in the medical notes, and communicating them to the ward nurses. Such decisions should follow locally agreed criteria based on knowledge of pre-arrest characteristics in relation to outcome. Patients' suitability for resuscitation should be reviewed on every consultant ward round, taking into account the views of all the staff caring for the patient and, when appropriate, the views of the patient or the patient's relatives, or both.
We thank the nurses and junior doctors at Fazakerley Hospital for filling in the questionnaires, and Dr F J Nye for his comments on the manuscript.

1 Keatinge RM. Exclusion from resuscitation. $f R$ Soc Med 1989;82:402-5.

2 Urburg $M$, Ways C. Survival after cardiopulmonary resuscitation for an in-hospital cardiac arrest. I Fam Pract 1987;25:41-4.

3 Bedell SE, Delbanco TL, Cook EF, Epstein FH. Survival after CPR in the hospital. NEnglf Med 1983:309:569-76.

4 Peatfield RC, Sillett RW, Taylor D, McNicol MW. Survival after cardiac arrest in hospital. Lancet 1977; i: 1223-5.

5 Asplund $K$, Britton $M$. Do-not-resuscitate orders in Swedish medical wards. f Intern Med 1990;228:139-45.

6 United Kingdom Central Council for Nursing, Midwifery and Health Visiting. Code of professional conduct for the nurse, midwife and health visitor. 2nd ed. London: UKCC, 1984.

Halligan M, Hamel RP. Ethics committee develops supportive care guidelines. Health Progress 1985;66:26-30,60.

8 Gleeson $\mathrm{K}$, Wise S. The do-not-resuscitate order: still too little too late Arch Intern Med 1990;150: 1057-60.

9 Kamer RS, Dieck EM, McClung JA, White PA, Sivak SL. Effect of New York State's do-not-resuscitate legislation on in-hospital cardiopulmonary resuscitation practice. Am f Med 1990;88:108-11.

10 McClung JA, Kamer RS. Implications of New York's do-not-resuscitate law. N Engl f Med 1990;323:270-2.

11 Stolman CJ, Gregory JJ, Dunn D, Levine JL. Evaluation of patient, physician, nurse and family attitudes toward do-not-resuscitate orders. physician, nurse and family a

12 Dent T, Gillard JH, Aarons EJ, Crimlisk H, Smyth-Pigott P. Preregistration $\omega$ house officers in the four Thames regions: I. Survey of education and workload. BMF 1990;300:713-6.

13 Wanzer SH, Federman DD, Adelstein SJ, Cassel CK, Cassem EH, Cranford $\infty$ $\mathrm{RE}$, et al. The physician's responsibility toward hopelessly ill patients. $\vec{\nabla}$ A second look. $N$ Engl f Med 1989;320:844-9.

(Accepted 3 October 1991)

\title{
Parathyroid hormone related protein and hypercalcaemia in breast cancer
}

\author{
N J Bundred, Wendy A Ratcliffe, Rosemary A Walker, S Coley, J M Morrison, J G Ratcliffe
}

Abstract

Objective-To see whether parathyroid hormone related protein has a humoral role in breast cancer.

Design-Plasma concentrations and tumour expression of parathyroid hormone related protein were determined (by two site immunoradiometric assay and immunohistochemistry respectively) in women with breast cancer and related to the presence of bone metastases and serum calcium concentrations.

Subjects-Plasma concentrations of parathyroid hormone related protein were measured in 57 women with early breast cancer without apparent bone metastases, 28 women with bone metastases, and 13 women with bone metastases and hypercalcaemia. Tissue positivity for parathyroid hormone related protein was determined retrospectively in 106 primary breast tumours from women without apparent bone metastases and 72 tumours from women with bone metastases, 25 of whom subsequently developed hypercalcaemia.

Results-Plasma parathyroid hormone related protein concentrations were detectable $(>0.23$ pmol/1) in $12(92 \%)$ of the 13 hypercalcaemic patients with bone metastases compared with $10(36 \%)$ of the 28 normocalcaemic patients with bone metastases and five $(9 \%)$ of the 57 normocalcaemic patients without bone metastases. Parathyroid hormone related protein concentrations were significantly higher in hypercalcaemic than normocalcaemic patients with bone metastases. Tumour staining was positive for parathyroid hormone related protein in $22(88 \%)$ of the 25 primary breast cancers from patients with bone metastases who later developed hypercalcaemia compared with $25(53 \%)$ of the 47 from women in this group who remained normocalcaemic and 55 (52\%) of the 106 early breast cancers from women without known metastases.
Conclusion-Tumour derived parathyroid hormone related protein may have an important humoral role in hypercalcaemia associated with metastatic breast cancer.

\section{Introduction}

Hypercalcaemia is a common complication of malig-o nancy. In patients with breast cancer it has traditionally been attributed to skeletal metastases and local osteolysis by tumour derived factors such as interleukin 1 , prostaglandins, and tumour necrosis factor. ${ }^{12} \frac{}{3}$ There is little evidence that these factors cause hypercalcaemia when introduced by injection, and it is $ᄋ$ assumed that their actions are largely paracrine, involving localised destruction of bone. The bio- 9 chemical changes seen in some patients with hypercal- N caemia and breast cancer are well documented and $D$ include increased renal tubular reabsorption of calcium, a decrease in the renal tubular threshold for $\bar{N}$ phosphate reabsorption, and increased excretion of N nephrogenous cyclic AMP. ${ }^{34}$ These abnormalities are consistent with the actions of a tumour derived parathyroid hormone-like factor with effects on both $\stackrel{0}{\odot}$ kidney and bone and have led to the hypothesis that the of mechanism of hypercalcaemia in breast cancer is mediated humorally by a parathyroid hormone-like $\overrightarrow{0}$ peptide. $^{45}$

Parathyroid hormone related protein is a hypercal- $\frac{}{\mathbb{Q}}$ caemic factor with similar bioactivity to that of para- 응 thyroid hormone. Since its isolation in 1987 from cancer cell lines and a carcinoma of the breast ${ }^{6}{ }^{6}$ strongo evidence has accumulated that parathyroid hormonerelated protein is an important humoral mediator ofo cancer associated hypercalcaemia in patients with solid $\risingdotseq$ tumours, typically squamous carcinoma of lung. ${ }^{78}$

We have investigated the possibility that parathyroid hormone related protein has a humoral role in 